\title{
Original article (short paper) \\ Influence of a virtual reality-based exercise protocol on the sit-to-stand activity kinematic variables in pregnant women: a randomized controlled trial
}

\author{
Silvia Oliveira Ribeiro \\ Vanessa Patricia Soares de Sousa \\ Elizabel de Souza Ramalho Viana \\ Universidade Federal do Rio Grande do Norte, Natal, RN, Brazil
}

\begin{abstract}
Aims: Changes resulting from the gestational period may lead to changes in the biomechanics of women, which can alter the performance of functional activities such as sit-to-stand. Thus, the objective of this study was to investigate the influence of a virtual reality-based exercise protocol on the kinematic variables of the sit-to-stand movement in women in their second and third gestational trimesters. Methods: The sample consisted of 44 women selected according to the eligibility criteria, allocated into 4 groups: control group, $2^{\text {nd }}$ trimester (CG2T); experimental group, $2^{\text {nd }}$ trimester $(\mathrm{EG} 2 \mathrm{~T})$; control group, $3^{\text {rd }}$ trimester $(\mathrm{CG} 3 \mathrm{~T})$; and experimental group, $3^{\text {rd }}$ trimester $(\mathrm{EG} 3 \mathrm{~T}) . \mathrm{All}$ the volunteers answered the identification and evaluation form and were sent to the kinematic evaluation through the Qualisys Motion Capture System ${ }^{\circledR}$. An intervention with game therapy was performed in 12 sessions of 30 minutes each, three times a week. Results: No statistically significant differences were found intra- $(\mathrm{P}>0.54)$ and inter-groups $(\mathrm{P}>0.059)$ for kinematic variables. However, there was a tendency for improvement in the analyzed variables after the proposed protocol. Conclusions: The data obtained suggest that the use of the Nintendo Wii Fit Plus ${ }^{\circledR}$ was not able to influence sit-to-stand kinematic variables in the analyzed women.
\end{abstract}

Keywords: biomechanical phenomena. pregnancy. gestacional age. exercise

\section{Introduction}

The various hormonal, physiological and anatomical changes that occur during pregnancy can result in changes in the biomechanics of women, capable of altering their balance ${ }^{1}$, postural control ${ }^{2-4}$ and their performance in carrying out functional activities ${ }^{5,6}$ such as sit-to-stand.

The ability to stand up from a sitting position imposes challenges on postural and motor control. According to Nicholls ${ }^{7}$, women in the third trimester report that the most difficult activities in this period are: sit-to-stand, picking up objects on the floor, seated work, driving, and getting out of the car. Limiting factors for these activities include postural instability, lumbopelvic pain, and fatigability ${ }^{7}$.

The sit-to-stand (STS) movement is operationally defined as a successful transfer of the body's center of mass (CM) from a sitting position where there is a large support base (thighs and feet) to a stable standing position ${ }^{8,9}$, followed by obtaining orthostatic balance when body oscillation must be controlled (stabilization phase) ${ }^{10-15}$. Therefore, it requires good coordination between the central nervous system (CNS) and the neuromuscular system ${ }^{8,9}$.

Performing the action of going from a sitting to a standing position is flexible and varies according to adaptation to the task requirements, the individual and the environment. However, studies that attempt to analyze this movement from the perspective of the daily routine of the population of pregnant women are still scarce. In this sense, the objective of this study was to investigate the influence of a virtual reality-based exercise protocol on STS movement kinematic variables of women in their second and third gestational trimesters. We adopted the position that virtual reality-based exercises would influence STS movement kinematic variables of pregnant women as the hypothesis.

\section{Material and methods}

This study is a randomized controlled clinical trial developed at the Laboratory of Intervention and Movement Analysis (LIAM) of the Physiotherapy Department of the Federal University of Rio Grande do Norte - UFRN, between April, 2014 and May, 2015.

The study population consisted of women in their second $\left(14^{\text {th }}\right.$ to $26^{\text {th }}$ week) and third (between 27 and 37 weeks) gestational trimester, living in the city of Natal-RN and participating in the Preparatory Course for Gestation, Childbirth and Postpartum $(C P G P P)$, promoted by the Physiotherapy Department of UFRN. The total population enrolled in the $C P G P P$ was 150 women during the study period through a non-probabilistic sampling process. The study sample consisted of 44 women allocated into 4 groups: Control Group - $2^{\text {nd }}$ Trimester (CG2T), Experimental Group - $2^{\text {nd }}$ Trimester (EG2T), Control Group - $3^{\text {rd }}$ Trimester (CG3T) and Experimental Group - $3^{\text {rd }}$ Trimester (EG3T).

The volunteers from Control Group (CG) participated in educational lectures (CPGPP) and those in the Experimental Group (EG) were additionally submitted to the exercise protocol using Wii-Rehabilitation, according to the flow chart below (Figure 1). 


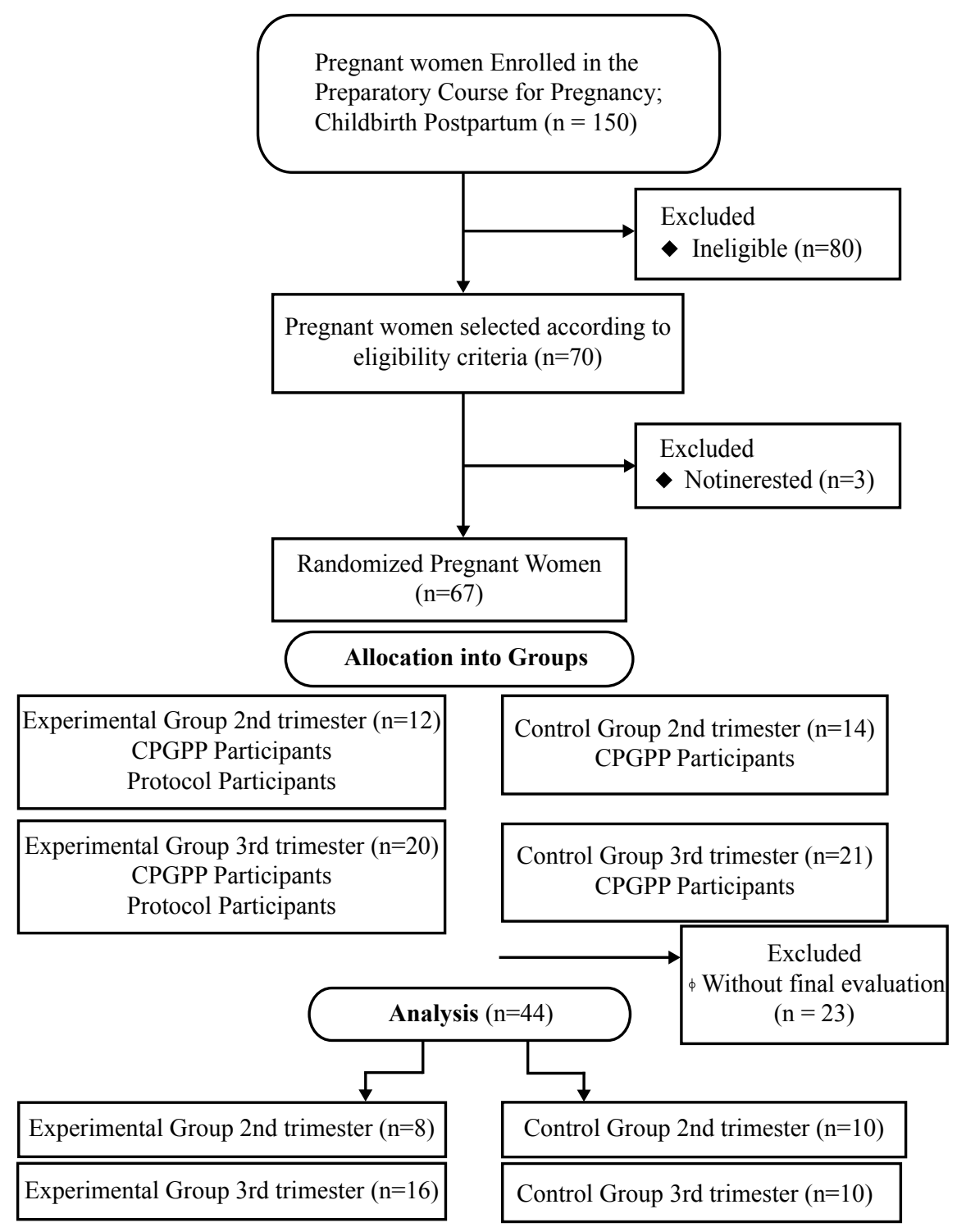

Figure 1. Study distribution flowchart according to CONSORT

The inclusion criteria were: (1) no clinical or obstetric changes (low-risk pregnancy); (2) being between 18 and 37 years of age; (3) being in the second or third gestational trimesters confirmed by ultrasonography, and between 14 to 21 weeks or 27 to 32 weeks at the initial evaluation; (4) being nulliparous; (5) attending prenatal care and having medical clearance to practice physical activity; (6) not using any medication/substances that affect balance; (7) self-report of no history of changes in balance before pregnancy; (8) absence of previous surgeries in the spine, pelvis, hip and knee; (9) no history of epilepsy or photosensitivity; and (10) no musculoskeletal, cardiorespiratory or neurological disorders that would impede accomplishing the evaluation and treatment protocols.

\section{Produceres}

The project was submitted to the Research Ethics Committee for Human Subjects of UFRN, approved under the opinion $n^{\circ} 719.939$, and registered in the Brazilian Registry of Clinical Trials under the Registry number RBR-4j35g5. All the volunteers signed the Free and Informed Consent Form (ICF) in accordance with the provisions of Resolution 466/12 of the National Health Council.

All participants were initially evaluated regarding sociodemographic, clinical, anthropometric and obstetric information. The volunteers were subsequently sent to kinematic evaluation (cinemetry), through a photogrammetry system based on video - Qualisys Motion Capture System ${ }^{\circledR}$ (QUALISYS MEDICAL AB, 41113 Gothenburg, Sweden), which enables evaluating kinematic parameters with angular, spatial and temporal data. The system consists of 8 cameras (Qualisys Oqus 300) and is based on three-dimensional (3D) reconstruction of passive (reflective) markers, positioned on specific bony prominences that delineate body segments.

To enable marker tracking and 3D data transformation, it is necessary for the system to recognize each camera's positioning and orientation, as well as the volume where the data will be captured. In this sense, system calibration process was performed 
through an L-shaped reference metal structure, positioned in the center of the walkway. This structure is composed of reflective markers located on the two axes (two on the shortest axis - X axis, and three on the longest axis - $\mathrm{Y}$ axis), enabling definition of the reference coordinates, represented by the $\mathrm{X}$ (mid-lateral), $\mathrm{Y}$ (anteroposterior) and $\mathrm{Z}$ axes (near-distal).

Spherical passive markers with 19 and $15 \mathrm{~mm}$ diameter were used in this study. Error prediction and maximum residual parameters were determined at $15 \mathrm{~mm}$ and $5 \mathrm{~mm}$, respectively. 2D data capture was performed by the Qualisys Track Manager 2.6 - QTM acquisition software, at a frequency of $120 \mathrm{Hertz}$ $(\mathrm{Hz})$. Next, the data generated in the QTM was exported to Visual 3D processing software (VISUAL3D Standard, 4.75.33 - CMotion, Rockville, MD, USA). This software is responsible for building the individual's biomechanical model, which allows for analyzing the spatial-temporal variables (velocity and total duration of movement) and angular variables range of motion of the trunk, hip, knee and ankle/foot complex $)^{16}$.

In order to delineate the segments and build the biomechanical model for analysis, reflexive markers were used in the following anatomical marks: C7; Trunk (acromion, bilaterally); Jugular incisures; Pelvis (highest point of the iliac crest, bilaterally); Thigh (greater trochanter, bilaterally; lateral epicondyle and medial femoral, bilaterally); Leg (lateral and medial malleolus, bilaterally); and foot (first and fifth metatarsal heads and distal end of the calcaneus, bilaterally) ${ }^{17}$. Trace markers were placed in the following locations: Pelvis (square-shaped plaque fixed at the right and left postero-superior iliac spine (PSIS) level using a Velcro fastened elastic band); Thigh (Rectangular shaped plate placed in the middle of the thigh fixed around the limb by velcro, bilaterally); Leg (Rectangular shaped plate placed in the middle of the thigh, fixed around the limb by velcro, bilaterally); Foot (in the aforementioned bony prominences - lateral malleolus, fifth metatarsal head and distal calcaneal extremity).

Thus, the marks defined the trunk, pelvis, thigh, leg and foot segments. The static position (reference position) analysis was performed with the volunteer standing, keeping their arms crossed on their chest and their feet apart for 3 seconds.

The STS movement was performed with the volunteer sitting on two blocks of wood without a backrest. The knees were flexed at 90 degrees and the feet were 10 centimeters apart at the heels ${ }^{18}$. The pregnant woman was instructed to "keep her arms crossed on her chest, stand up securely without support and at her comfortable speed, without changing the positioning of the feet". The volunteers performed the movement twice in order to confirm assimilation, correct positioning and safety. Five data collections were subsequently carried out for recording.

\section{Reduction of Kinetic analysis data}

After data capturing and processing on the Qualisys Track Manager (QTM, version 2.6) with the named markers and their defined trajectories, study movement cycles were selected in order to allow frame interpolation in each of these cycles.

Next, data processed in the QTM were exported to the Visual $3 D$ software. For this, static marker position and anthropometric data (height and weight) of the participant were used in the collection. For data reduction and analysis, three of the five performed tests were selected ${ }^{17}$. The tests chosen for analysis were the most homogeneous, meaning those that had values close to the average/mean generated from the 5 tests.

Each model segment was defined through an association of anatomical marks, and arranged sequentially: the markers attached to the medial portion of the iliac crest, greater trochanter of the femur and the cluster positioned at the sacrum base define the pelvis segment; the anatomical marks of the major trochanter, lateral and medial epicondyle of the femur, associated with the cluster positioned on the lateral side and the middle third of the thigh define the thigh segment; while the leg segment is defined by the lateral and medial epicondyle of the femur, lateral and medial malleoli of the ankle, along with the cluster positioned on the side and middle third of the leg. The ankle-foot complex is defined by the markers located on the lateral and medial malleoli, calcaneus and on the $1^{\text {st }}$ and $5^{\text {th }}$ metatarsal heads ${ }^{17}$.

To eliminate the noises caused by marker movement, a low pass filter (Low Pass Butterworth) with the cutoff frequency set at $6 \mathrm{HZ}$ to the marker trajectories was applied ${ }^{19}$.

Each angular displacement was obtained by associating the segments with a coordinate system that uses a sequence of the Cardan angles, defined as the coordinate system orientation of a segment in relation to a reference coordinate system ${ }^{20}$. In order to characterize the beginning of the sitting to standing motion, the onset moment of trunk movement was recorded considering the first anterior displacement of the CV7 marker. In order to characterize the end of the movement, we considered the moment in which horizontal displacement of marker CV7 (axis y; sagittal plane) remained stable, forming a plateau (from the moment that the horizontal values of marker $\mathrm{CV} 7$ remained the same, after three frames of movement) and the individual reached the upright position ${ }^{17}$.

The following events were defined to determine the beginning and end of the studied movement and the phases of this movement ${ }^{17}$ :

- Initial Movement (IM): Moment at the beginning of trunk movement observed by the first anterior displacement of CV7 marker.

- Maximal ankle dorsiflexion (Max AD): Moment the ankle reached maximum dorsiflexion.

- End of extension (EXT): End of hip extension, accompanied by the end of trunk and knee extension.

- Final Movement (FM): the moment that CV7 marker remained stable and the individual reached an erect position.

Three phases for movement were defined for this study using previously identified events, namely:

- Flexion phase (P1): from the Initial Movement (IM) until maximal dorsiflexion of the ankle (Max AD).

- Extension phase (P2): from Max AD until the end of hip extension, accompanied by the end of trunk and knee extension (EXT). 
- Stabilization phase (P3): from EXT until the moment that CV7 marker remained stable and the individual reached an erect position (FM).

\section{Intervention Protocol}

The intervention with the virtual reality program was developed in twelve (12) sessions lasting thirty (30) minutes, not including rest time which was about 2 minutes rest after each game. The frequency was three (3) times per week for a period of four (4) weeks. All participants were instructed not to do the Nintendo Wii Fit Plus ${ }^{\circledR}$ balance exercises at home.

Individual sessions were conducted with a Wii balance board ${ }^{\circledR}$ (WBB), recently validated as a strength platform (20) and as an instrument for equilibrium analysis in the orthostatic position (2).

In the virtual training environment there was a television connected to a Wii console, equipped with sensors responsible for obtaining the data sent by the balance board and controls via wireless transfer. The balance board was placed directly on the floor at a distance of 2.4 meters from the TV; a physiotherapist was constantly positioned next to the volunteer, being responsible for providing guidance and monitoring the participant throughout the duration of the intervention.

The Wii Fit Plus ${ }^{\circledR}$ package games used for the balance training were: Balance bubble, Tightrope, Ski jump, Penguin slidee and Soccer heading. All participants engaged in five games in the order described above, with designated time of 4 minutes for each set, corresponding to approximately to 2 cycles. The volunteers had one familiarization session with the games.

\section{Statistical Analysis}

Initially, descriptive statistics from sociodemographic, clinical, anthropometric and obstetric variables were conducted with the objective of characterizing the sample through measures of central tendency, dispersion, absolute and relative values.

In order to verify the interaction between the groups, $4 \times 2$ repeated measures ANOVA test was used adopting time factors and analysis groups for the cinemetry variables. Next, the mean of the obtained values from the three tests selected for each individual was obtained for kinematic variable data analysis. A significance level of $\mathrm{P}<0.05$ was used.

\section{Results}

Sample characterization regarding sociodemographic, obstetric and anthropometric variables at the time of the initial evaluationis shown in Table 1.

Table 2 shows the instant of occurrence (in seconds) of the events in the sit-to-stand (STS) movement in the four groups evaluated from the initial and final evaluations.

When considering the STS movement events, no statistically significant differences were found for intra or intergroup conditions (IM- intragroup $[\mathrm{F}=0.06 ; \mathrm{P}=0.79]$; intergroup $[\mathrm{F}=0.30 ; \mathrm{P}=0.82] ; \mathbf{E X T m a x}-$ intragroup $[\mathrm{F}=1.52 ; \mathrm{P}=0.69]$; intergroup $[\mathrm{F}=0.11 ; \mathrm{P}=0.94] ; \mathbf{F M}$ - intragroup $[\mathrm{F}=2.20 ; \mathrm{P}=0.14]$; intergroup $[\mathrm{F}=0.41 ; \mathrm{P}=0.74])$. Table 3 shows the time (in seconds) of the STS movement phases and the phase time percentage in relation to the total time of the movement in the initial and final evaluation.

When considering the STS movement phases and total duration, no statistically significant differences were found for intra and intergroup conditions ( $\mathbf{P 1}$ - intragroup $[\mathrm{F}=0.140 ; \mathrm{P}=$ 0.71]; intergroup $[\mathrm{F}=2.69 ; \mathrm{P}=0.059] ; \mathbf{P} 2$ - intragroup $[\mathrm{F}=1.96 ; \mathrm{P}=$ $0.16]$; intergroup $[\mathrm{F}=0.127 ; \mathrm{P}=0.94] ; \mathbf{P} 3$ - intragroup $[\mathrm{F}=0.329$; $\mathrm{P}=0.57]$; intergroup $[\mathrm{F}=0.743 ; \mathrm{P}=0.53]$; Total Time - intragroup $[\mathrm{F}=0.365 ; \mathrm{P}=0.54]$. Intergroup $[\mathrm{F}=0.303 ; \mathrm{P}=0.82]$ ).

Regarding the comparison of the angular displacement of the hip joint between the IM and Extmax in the STS movement, no statistically significant difference was observed in intra and intergroup conditions ( $\mathbf{R O M}$ hip) - intragroup $[\mathrm{F}=1.09 ; \mathrm{P}=0.30]$; intergroup $[\mathrm{F}=0.37 ; \mathrm{P}=0.77])$.

Figure 2 shows the mean angular displacement of the hip joint in the 4 study groups: CG2T, EG2T, CG3T and EG3T. Hip flexion is observed in the STS movement, followed by the extension of this joint. Regarding comparison of the angular displacement of the hip joint ROM, no statistically significant difference was found between the analyzed groups.

Table 1. Sociodemographic, obstetrical and anthropometric characteristics of pregnant women participating in the study at the initial evaluation

\begin{tabular}{lcc}
\hline \multicolumn{1}{c}{ Variables } & $\mathbf{2 T}(\mathbf{n}=\mathbf{1 8})$ & $\mathbf{3 T}(\mathbf{n = 2 6 )}$ \\
\hline Sociodemographic data & & \\
\hline Age (in years) & $28.55 \pm 3.83$ & $29.42 \pm 3.23$ \\
Education level (in years) & $16.97 \pm 2.58$ & $16.26 \pm 4.18$ \\
Marital status & $61.1 \%(11)$ & $84.6 \%(22)$ \\
Married & $33.3 \%(6)$ & $15.4 \%(4)$ \\
Has a partner & $5.6 \%(1)$ & - \\
Does not have a partner & & \\
\hline Obstetrical data & $18.83 \pm 3.11$ & $29.32 \pm 2.32$ \\
\hline GA (in weeks) & & \\
\hline Anthropometric data & $68.91 \pm 11.83$ & $68.12 \pm 7.90$ \\
\hline Weight (in kg) & $1.64 \pm 5.87$ & $1.62 \pm 6.29$ \\
Height (in meters) & & \\
BMI* & $16.7 \%(3)$ & $76.9 \%(20)$ \\
Adequate & $83.3 \%(15)$ & $23.1 \%(6)$ \\
Overweight & $66.7 \%(12)$ & $57.7 \%(15)$ \\
Obese & $22.2 \%(4)$ & $42.3 \%(11)$ \\
\hline Low Back Pain & $11.1 \%(2)$ & - \\
\hline Yes & & \\
No & & \\
\hline & & \\
\hline
\end{tabular}


Table 2. Comparison of the occurrence instant of STS events considering the two moments of evaluation between the analyzed groups

\begin{tabular}{|c|c|c|c|c|c|c|c|}
\hline \multirow[b]{2}{*}{ Events } & \multirow[b]{2}{*}{ Group } & \multicolumn{2}{|r|}{ Before } & \multicolumn{2}{|r|}{ After } & \multicolumn{2}{|c|}{ 95\% Confidence Interval } \\
\hline & & Mean (s) & Standard Deviation \pm & Mean (s) & Standard Deviation \pm & Lower bound & Upper bound \\
\hline \multirow{4}{*}{ IM } & CG2T & 1.02 & 0.51 & 0.99 & 0.32 & \multirow{4}{*}{0.974} & \multirow{4}{*}{1.23} \\
\hline & EG2T & 1.11 & 0.67 & 1.01 & 0.48 & & \\
\hline & CG3T & 1.13 & 0.33 & 1.17 & 0.36 & & \\
\hline & EG3T & 1.09 & 0.56 & 1.20 & 0.40 & & \\
\hline \multirow{4}{*}{ Extmax } & CG2T & 1.38 & 0.5 & 1.39 & 0.40 & \multirow{4}{*}{1.38} & \multirow{4}{*}{1.65} \\
\hline & EG2T & 1.51 & 0.70 & 1.40 & 0.44 & & \\
\hline & CG3T & 1.60 & 0.37 & 1.60 & 0.37 & & \\
\hline & EG3T & 1.68 & 0.52 & 1.62 & 0.46 & & \\
\hline \multirow{4}{*}{ FM } & CG2T & 1.68 & 0.61 & 1.69 & 0.47 & \multirow{4}{*}{1.65} & \multirow{4}{*}{1.93} \\
\hline & EG2T & 1.88 & 0.74 & 1.59 & 0.48 & & \\
\hline & CG3T & 1.99 & 0.37 & 1.83 & 0.47 & & \\
\hline & EG3T & 1.88 & 0.58 & 1.76 & 0.57 & & \\
\hline
\end{tabular}

NOTE: Values are expressed as mean \pm Standard Deviation. $4 \times 2$ ANOVA for repeated measures test was used. LEGEND: IM - initial movement; Extmax Maximum hip extension; FM - Final movement; CG2T - Control Group 2nd trimester; EG2T - Experimental Group 2nd trimester; CG3T - Control Group 3rd trimester; EG3T - Experimental Group 3rd trimester.

Table 3. Comparison of the phase duration and the total time of the movement in seconds considering the two evaluation moments between the analyzed groups

\begin{tabular}{|c|c|c|c|c|c|c|c|c|c|}
\hline \multirow[b]{2}{*}{ Events } & \multirow[b]{2}{*}{ Group } & \multicolumn{3}{|c|}{ Before } & \multicolumn{3}{|c|}{ After } & \multicolumn{2}{|c|}{ 95\% Confidence Interval } \\
\hline & & Mean (s) & $\begin{array}{c}\text { Standard } \\
\text { Deviation } \pm\end{array}$ & $\begin{array}{l}\text { Phase } \\
\text { time \% }\end{array}$ & Mean (s) & $\begin{array}{c}\text { Standard } \\
\text { Deviation } \pm\end{array}$ & $\begin{array}{l}\text { Phase } \\
\text { time \% }\end{array}$ & $\begin{array}{l}\text { Lower } \\
\text { bound }\end{array}$ & $\begin{array}{l}\text { Upper } \\
\text { bound }\end{array}$ \\
\hline \multirow{4}{*}{ P1 } & CG2T & 0.64 & 0.25 & 27.35 & 0.68 & 0.34 & 29.95 & \multirow{4}{*}{0.557} & \multirow{4}{*}{0.702} \\
\hline & EG2T & 0.55 & 0.25 & 21.48 & 0.62 & 0.38 & 25.94 & & \\
\hline & CG3T & 0.76 & 0.20 & 28.89 & 0.55 & 0.28 & 21.23 & & \\
\hline & EG3T & 0.52 & 0.29 & 19.40 & 0.68 & 0.27 & 24.72 & & \\
\hline \multirow{4}{*}{$\mathrm{P} 2$} & CG2T & 0.47 & 0.22 & 20.08 & 0.45 & 0.18 & 19.82 & \multirow{4}{*}{0.398} & \multirow{4}{*}{0.506} \\
\hline & EG2T & 0.46 & 0.19 & 17.96 & 0.40 & 0.18 & 16.73 & & \\
\hline & CG3T & 0.44 & 0.19 & 16.73 & 0.35 & 0.17 & 13.51 & & \\
\hline & EG3T & 0.53 & 0.27 & 19.77 & 0.48 & 0.22 & 17.45 & & \\
\hline \multirow{4}{*}{ P3 } & CG2T & 0.19 & 0.09 & 8.11 & 0.19 & 0.13 & 8.37 & \multirow{4}{*}{0.264} & \multirow{4}{*}{0.330} \\
\hline & EG2T & 0.19 & 0.08 & 7.42 & 0.34 & 0.43 & 14.22 & & \\
\hline & CG3T & 0.39 & 0.18 & 14.82 & 0.37 & 0.22 & 14.28 & & \\
\hline & EG3T & 0.33 & 0.29 & 12.31 & 0.31 & 0.20 & 11.27 & & \\
\hline \multirow{4}{*}{ Total Time } & CG2T & 2.34 & 0.96 & 100 & 2.27 & 0.55 & 100 & \multirow{4}{*}{2.32} & \multirow{4}{*}{2.73} \\
\hline & EG2T & 2.56 & 0.84 & 100 & 2.39 & 0.74 & 100 & & \\
\hline & CG3T & 2.63 & 0.70 & 100 & 2.59 & 0.86 & 100 & & \\
\hline & EG3T & 2.68 & 0.54 & 100 & 2.75 & 0.60 & 100 & & \\
\hline
\end{tabular}

NOTE: Values are expressed as mean \pm Standard Deviation. 4x2 ANOVA for repeated measures test was used. LEGEND: P1 - flexion phase; P2 - extension phase; P3, stabilization phase; CG2T - Control Group 2nd trimester; EG2T - Experimental Group 2nd trimester; CG3T - Control Group 3rd trimester; EG3T Experimental Group 3rd trimest 

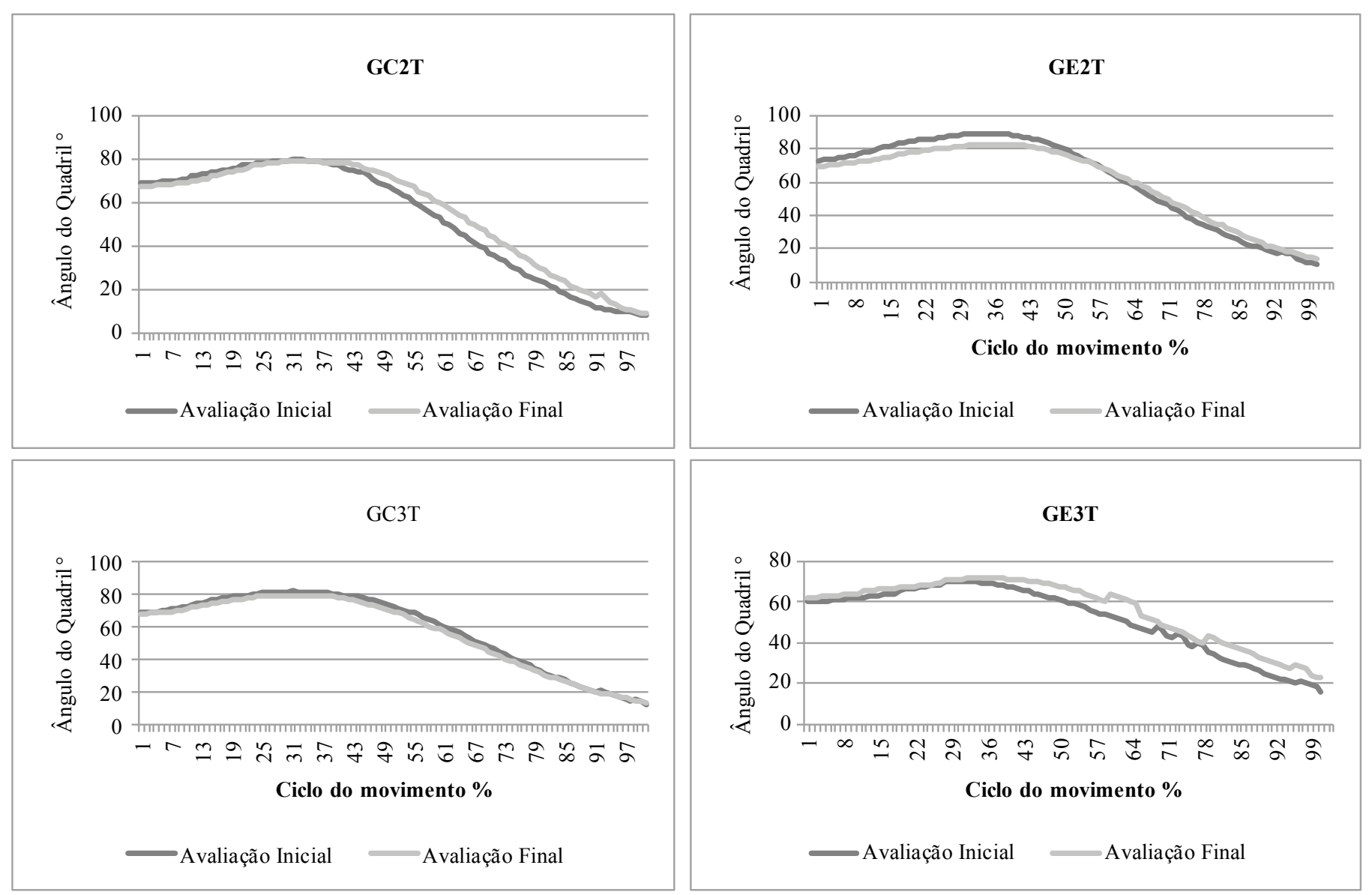

Figure 2. Angular displacement means of the hip joint during the sit-to-stand cycle

\section{Discussion}

The STS movement requires that adequate torque is developed at each joint ${ }^{21}$. The difficulty of moving from sitting to standing on both feet requires the use of several strategies in order to facilitate its execution. When older individuals perform this movement, for example, there is an increase in postural stability by the use of the upper limbs as support. This adaptation allows the mass center of the support surface to be approached by increased torso flexion or time spent in performing the movement. These strategies result in increased energy expenditure in this population as a result of the higher recruitment of motor units ${ }^{22}$.

The difficulty in performing the movement may also be related to physiological factors, the initial position of the body segments and environmental factors. Physiological factors include decreased proprioceptive acuity, muscle strength, postural balance deficit, and joint pain, limiting the range of motion ${ }^{22}$.

Factors such as seat height, depth and inclination/slope of the seat can also make STS more difficult. Increased seat height, for example, decreases the muscular force required to perform the movement. This increase may result in new biomechanical requirements (such as the need to move the body center of mass at a greater distance) or an altered strategy (such as the "stabilization strategy"). This is due to the biomechanical requirements imposed by the foot, torso, or arm position ${ }^{10,15}$. However, Lou,
Chou, Chou, Lin, Chen ${ }^{23}$ observed that increasing the time to perform the STS movement has become more significant for seats with lower heights ${ }^{23}$.

In our study we chose to use two wooden blocks (Balance Master ${ }^{\circledR}$ accessories) without backrest, keeping the knees bent at 90 degrees and the feet 10 centimeters apart at the heels ${ }^{18}$.

Studies that analyze STS tend to restrict the use of the arms for performing the movement. Individuals are advised to adopt the orthostatic position by keeping their hands in certain positions, such as: cradles at the level of the chest, arranged at the side of the body, or resting on the knees ${ }^{12,15}$. The presence of arm support is an important environmental strategy for the older adult population and also for pregnant women. Therefore we adopted the crossed arms on the chest position during kinematic analysis of the movement.

For Carr and Shepherd ${ }^{12}$, the restricted arms position during STS seemed to cause a different pattern of angular displacement of the ankle, with a much larger mean standard deviation than that of free arms ${ }^{12,15}$. The results of the study by Takeda, Katsuhira, Takano ${ }^{24}$ found that when an armrest is used, the existing reaction force facilitates a forward and upward center of gravity displacement. In addition, a symmetrical dispersion of the load on the legs occurs, thus minimizing the load placed on the feet. Therefore, this suggests that the use of an arm support would decrease the muscular load when performing the sit-to-stand activity ${ }^{24}$. 
The findings of Carr and Shepherd ${ }^{12}$ and Takeda, Katsuhira, $\mathrm{Takano}^{24}$ corroborate those by Lou, Chou, Chou, Lin, Chen ${ }^{23}$, who stated that it is useful to instruct pregnant women to adopt relatively higher-seating chairs and to use the armrests to help ensure postural stability and avoid putting pressure on the abdomen ${ }^{23}$. This adaptation would also help to decrease the joint torque of the hips and knees when there is loss of contact with the seat ${ }^{22}$.

The movement under analysis includes flexion of the torso and extension of the back, hips and knees, made difficult by painful symptoms in the lower back and/or extremities ${ }^{23}$. As pregnancy progresses, maximum torso flexion is reduced and the body mass is increased as a result of the impregnated uterus. In addition, hip joint peak and flexion speed decrease ${ }^{23,25}$. Therefore, it is expected that with gestation advancement, the activity of raising/lifting oneself from a chair becomes more difficult to perform. Consequently, the kinematic and kinetic variables would be altered as strategies to complete the task ${ }^{26}$.

In our results, we did not obtain significant differences for the STS kinematic movement variables when compared to the analysis groups. Likewise, the findings by Takeda Katsuhira, Takano ${ }^{24}$ did not find any differences for STS movement between the gestational trimesters ${ }^{24}$. This suggests that pregnant women seem to be aware of postural instability during functional activities and the fear of falls seems to make them more cautious. This fact could cause the propulsion phase to be minimized, and as a consequence, the extension phase duration ${ }^{25}$. However, no significant differences were observed between the analyzed groups $(\mathrm{P}=0.94)$ for this variable.

Savelberg, Fastenau, Willems, Meijer ${ }^{27}$ investigated the influence of increased load on the movement of rising from a chair in healthy young women. The volunteers performed the movement using a vest with an additional $0 \%, 15 \%, 30 \%$ and $45 \%$ of their body mass. The authors observed that in the $45 \%$ condition, the movement lasted on average $22 \%$ longer than in the $0 \%{ }^{27}$. Thus, we expected that in the third trimester of gestation, pregnant women would present a longer total STS movement duration. However, the total duration of STS movement between groups did not differ significantly $(\mathrm{P}=0.82)$. It is necessary, however, to take into account that the mass distribution added to the volunteers' bodies of the study by Savelberg Fastenau, Willems, Meijer ${ }^{27}$ may have been different from the physiological distribution of weight gain that happens in pregnant women.

Mazzà, Benvenuti, Bimbi, Stanhope ${ }^{10}$ suggests that the higher degree of difficulty of a given task associated with the functional status of the individual influences the compensatory strategies that will be used when raising oneself from a chair ${ }^{10}$. Such claims can justify the absence of difference for this variable when analyzing the groups of pregnant women. The need to control the upright balance at the end of the climb is paramount, and participants may have voluntarily limited the propulsion momentum to maintain upright posture under varying conditions. In this context, the obtained result suggests that the pregnant women sought to perform a more weighted movement ${ }^{26}$.

\section{Conclusion}

To our knowledge, this study is the first to be conducted using virtual reality to observe its influence on the sit-to-stand movement kinematics in pregnant women. Although the hypothesis of this study (that the virtual reality-based exercise protocol would influence the kinematic variables of pregnant women during the sit-to-stand) was not confirmed, it is important to emphasize the absence of studies that analyze the effect implementing virtual reality games on the biomechanics of the sit-to-stand movement in pregnant women.

The non-confirmation of the hypothesis of this study can be justified by: 1) the responses produced by the volunteers seem to have been more sensitive to the adaptation of sensory systems and the integration between them than to the biomechanical alterations to which the woman's body is subjected; and 2) changes in the biomechanics of pregnant women suggest adaptations in order to minimize the limitation effects, thus increasing stability during movement.

The contradictions between the studies regarding the analysis instruments of the sit-to-stand movement kinematics, along with the scarcity of research that describe the influence of physiological, hormonal and anatomical changes on the postural control of pregnant women evidence the need for further studies analyzing postural control pattern in pregnancy during everyday activities.

One of the limitations of the study was the small sample number and the non-accomplishment of follow-up. In addition, the intervention time may have been insufficient so that the answers promoted by the training with virtual reality could be acquired and automated, enabling the transfer of this motor task from the games to execution of the movement during the evaluation. However, the results demonstrated in this study are important and justify further studies analyzing the influence of implementing virtual reality on cinemetry variables (occurrence of events and movement phase duration) in pregnant women.

\section{References}

1. Ersal T, McCrory JL, Sienko KH. Theoretical and experimental indicators of falls during pregnancy as assessed by postural perturbations. Gait Posture [Internet]. Elsevier B.V. 2014;39(1):218-23.

2. Jang J, Hsiao KT, Hsiao-Wecksler ET. Balance (perceived and actual) and preferred stance width during pregnancy. Clin Biomech (Bristol, Avon) [Internet]. 2008 May [cited 2013 Oct 21];23(4):468-76.

3. McCrory JL, Chambers AJ, Daftary A, Redfern MS. Dynamic postural stability in pregnant fallers and non-fallers. BJOG [Internet]. 2010;117(8):954-62.

4. Gazaneo MM, Oliveira LF. Alteração postural durante gestação. Rev bras ativ fís saúde. 1998;3(2):13-21.

5. Sunaga Y, Anan M, Shinkoda K. Biomechanics of rising from a chair and walking in pregnant women. Appl Ergon [Internet]. Elsevier Ltd; 2013;44(5):792-8.

6. Ribas SI, Guiro ECO. Análise da pressão plantar e do equilíbrio postural em diferentes fases da gestação. Rev Bras Fisioter. 2007;11(5):391-6. 
7. Nicholls JA.Performance of physical tasks in pregnancy. Ergonomics. 1992; 35(3): 301-11.

8. Ramsey VK, Miszko TA, Horvat M. Muscle activation and force production in Parkinson's patients during sit to stand transfers. Clin Biomech (Bristol, Avon) [Internet]. 2004;19(4):377-84.

9. Mathiyakom W, McNitt-Gray JL, Requejo P, Costa K. Modifying center of mass trajectory during sit-to-stand tasks redistributes the mechanical demand across the lower extremity joints. Clin Biomech (Bristol, Avon) [Internet]. 2005;20(1):105-11.

10. Mazza C, Benvenuti F, Bimbi C, Stanhope SJ. Association Between Subject Functional Status, Seat Height, and Movement Strategy in Sit-to-Stand Performance. JAm Geriatr Soc. 2004;52:1750-4.

11. Dehail P, Bestaven E, Muller F, Mallet A, Robert B, BourdelMarchasson I, et al. Kinematic and electromyographic analysis of rising from a chair during a "Sit-to-Walk" task in elderly subjects: role of strength. Clin Biomech (Bristol, Avon) [Internet]. 2007;22(10):1096-103.

12. Carr JH, Shepherd R. Reabilitação Neurológica: Otimizando o Desempenho Motor. $1^{\circ}$ edição. São Paulo: Editora: Manole, 1998, pag:74.

13. Mourey F, Grishin A, d'Athis P, Pozzo T, Stapley P. Standing up from a chair as a dynamic equilibrium task: a comparison between young and elderly subjects. J Gerontol A Biol Sci Med Sci [Internet]. 2000;55(9):425-31.

14. Riley P, Schenkman ML, Mann RW, Andrew W. Mechanics of a consttrained chair-rise. J Biomechanics. 1991;24(I): 77-85.

15. Janssen, Win GM, Hans BJ Bussmann HJS. Research Report Determinants of the Sit-to-Stand Movement : A Review. J Am Phys Ther Assoc Fysiotherapeut. 2002;82:866-79.

16. Visual. Visual3D Online Documentation C-Motion.

17. Lana C. Caracterização Cinemática da Atividade de Passar de Sentado para Andar em Indivíduos com Doença de Parkinson. Belo Horizonte. Dissertação [Mestrado]- Universidade de Belo Horizonte; 2010.

18. Brech GC, Alonso AC, Luna NMS, Greve JM. Correlation of postural balance and knee muscle strength in the sit-to-stand test among women with and without postmenopausal osteoporosis. Osteoporos Int [Internet]. 2013;24(7):2007-13.

19. Robertson DG, Dowling JJ. Design and responses of butterworth and critically dampted digital filters. J Electromyogr Kinesiol. 2003;13(6):69-73
20. Cole GK, Nigg BM, Ronsky JL, Yeadon MR. Application of the joint coordinate system to three-dimensional joint attitude and movement representation: a standardization proposal. J Biomech Eng.1993;115(4):344-9.

21. Schultz AB, Alexander N B, Ashton-Miller JA. Analyses of rising from a chair. J Biomech. 1992;25(12):1383-91

22. Goulart F, Chaves CM, Chagas MLD, Carvalho A, Saiki KR. O movimento de passar de sentado para de pé em idosos : implicações para o treinamento funcional. Acta fisiátrica. 2003;10(3):138-143

23. Lou S, Chou Y, Chou P, Lin C, Chen U. Sit-to-stand at different periods of pregnancy. Clin Biomech. 2001;16:194-8.

24. Takeda K, Katsuhira J, Takano A. Effects of handrail use during sit-to-stand in the third trimester. Int J Ind Ergon [Internet]. Elsevier Ltd; 2009;39(6):988-94.

25. Gilleard W, Crosbie J, Smith R. A longitudinal study of the effect of pregnancy on rising to stand from a chair. J Biomech [Internet]. 2008;41(4):779-87.

26. Gilleard W, Lai DT, Levinger P BR. Detecting trunk motion changes due to pregnancy using pattern recognition techniques. Eng Med Biol Soc. 2008;

27. Savelberg HH, Fastenau A, Willems PJ, Meijer K. The load/ capacity ratio affects the sit-to-stand movement strategy. Clin Biomech (Bristol, Avon). 2007;22(7):805-12.

\section{Corresponding author}

Silvia Oliveira Ribeiro

Postal Address: Department of Physical Therapy, Federal University of Rio Grande do Norte, Avenue Senador Salgado Filho, 3000, Natal, Rio Grande do Norte, Brazil. Email: oliveira.silviaribeiro@gmail.com

Manuscript received on May 1, 2017

Manuscript accepted on May 7, 2017

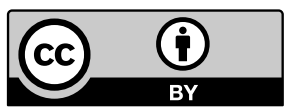

Motriz. The Journal of Physical Education. UNESP. Rio Claro, SP, Brazil - eISSN: 1980-6574 - under a license Creative Commons - Version 3.0 